\title{
Motor unit recruitment as reflected by muscle fibre glycogen loss in a prosimian (bushbaby) after running and jumping ${ }^{1}$
}

\author{
C. A. GILlESPIE, D. R. SIMPSON, AND V. REGGIE EDGERTON ${ }^{2}$ \\ From the Neuromuscular Research Laboratory and Brain Research Institute, \\ University of California, Los Angeles, California, U.S.A.
}

SYNOPSIS A qualitative histochemical assessment of glycogen loss in biopsies was made in bushbabies after running and jumping. Glycogen loss was related to the specific type of exercise. After running, glycogen loss was greatest in the slow-twitch oxidative fibre and depletion in the fast-twitch oxidative glycolytic fibres was similarly greater than in the fast-twitch glycolytic fibres. After jumping, the opposite pattern of glycogen utilization occurred $(\mathrm{FG}>\mathrm{FOG}>\mathrm{SO}$ ).

Although much is known about the dynamic, biochemical, and fatigue properties of the muscle fibre types, until recently virtually nothing was known about their functional significance and preferential recruitment in exercise. The loss of fibre glycogen, a substrate which serves as an important energy source for muscular contraction, has been used as an indication of fibre recruitment in exercise and single motor unit recruitment (Edgerton et al., 1970b; Burke et al., 1971; Edgerton and Hewitt, 1972b; Edgerton and Lehto, 1972c).

While the utilization of a given fibre type was consistent within each animal, not all rats and guinea-pigs displayed the same fibre selectivity when run on a treadmill (Edgerton et al., 1971). Although mice and rats utilized mainly fast oxidative-glycolytic fibres in swimming in preference to fast-glycolytic, glycogen loss in the guinea-pig was found selectively in fast glycolytic fibres. The reason for this variability was unknown (Edgerton et al., 1970b; Edgerton et al., 1971).

To gain a greater understanding of fibre

1 These data in part were presented to the Second Annual meeting for the Society of Neurosciences, Houston, 1972. The work was supported in part by USPHS NS-10497 and the Biomedical Sciences Support Grant, UCLA.

2 Address for correspondence: V. Reggie Edgerton, Department of Kinesiology, Neuromuscular Research Laboratory and Brain Research Institute, University of California, Los Angeles, California 90024, U.S.A. recruitment, two markedly different exercise tasks were studied in a non-human primate. Adult bushbabies (Galago senegalensis) were trained to run or jump on a treadmill by altering the speed of the treadmill. Specific fibre type involvement was examined in predominantly fast twitch (vastus lateralis and gastrocnemius) and slow twitch (soleus) muscles.

\section{METHOD}

Six adult lesser bushbabies (Galago senegalensis) were used to study fibre recruitment in predominantly fast and slow twitch muscles during continuous gait running and jumping. Animal care and maintenance has been described previously (Edgerton et al., 1972a).

The animals were run during their active diurnal cycle on a motor driven treadmill which was covered with a plexiglass housing as described previously by the same authors. For jumping, the treadmill was covered with a plexiglass housing $40 \mathrm{~cm}$ wide $\times 170 \mathrm{~cm}$ long $\times 60 \mathrm{~cm}$ high with a $10 \mathrm{~cm}$ drop at the rear to a $20 \mathrm{~cm}$ long shock grid. The $3.2 \mathrm{~mm}$ diameter brass rods of the grid were perpendicular to the direction of the movement of the belt, $3.2 \mathrm{~mm}$ apart, and supplied with d.c. electric charges of 20$80 \mathrm{~V}(2 \mathrm{~mA})$. Each animal was exposed to the exercise task on several occasions and their legs were shaved at the biopsy site before the day the experiment was begun. 
Individual Galagos ran $5-15 \mathrm{~min}$ at $1.75 \mathrm{~m} / \mathrm{min}$ or jumped $2-4 \mathrm{~min}$ at $2.4-2.9 \mathrm{~m} / \mathrm{min}$. Immediately after the exercise task the animal was anaesthetized by inhalation of methoxyflurane. Through lateral incisions, small biopsies (about $2 \times 2 \times 8 \mathrm{~mm}$ ) of one or more of the following muscles were taken: vastus lateralis (VL), gastrocnemius (G), and soleus (Sol).

One half of a muscle sample was frozen for histochemistry in isopentane which was cooled with liquid nitrogen. The other half was assayed biochemically for glycogen (Huijing, 1970). Histochemical biopsies were sectioned at $10 \mu \mathrm{m}$ and incubated for myosin adenosine triphosphatase with alkaline preincubation (Guth and Samaha, 1969), reduced nicotinamide adenine dinucleotide diaphorase (Novikoff et al., 1961 ), and $\alpha$-glycerophosphate dehydrogenase (Wattenberg and Leong, 1960). The same tissue blocks were subsequently cut at $20 \mu \mathrm{m}$ for the demonstration of glycogen employing the PAS technique (Pearse, 1961).

Photographs taken of entire areas of these stained cross-sections were used to classify the fibres as fasttwitch oxidative glycolytic (FOG), 'moderate' (M), fast-twitch glycolytic (FG), or slow-twitch oxidative (SO) (Edgerton and Simpson, 1969; Barnard et al., 1971; Peter et al., 1972). The moderate $(M)$ fibres of the fast-twitch fibre population represent the mid-range of the continuum of NADH-D staining intensity between FOG (dark) and FG (light). Glycogen depletion was analysed as the proportion of each fibre type that subjectively demonstrated dark (3), moderate (2), light (1), or negative (0) PAS staining intensity (Figs 1 and 3).

Percent weighted glycogen loss was calculated by multiplying the number of dark, moderate, light, and negative PAS staining fibres of each fibre type by 3 , 2,1 , and 0 respectively, summating these values and calculating the difference $(\%)$ between this value and the sum obtained when all fibres of a given sample were rated 3 (dark) (Figs 2 and 4).

\section{RESULTS}

Individual biopsy fibre populations are represented by the length of the bars in Figs 1, 2, 3, and 4. No SO fibres were found in VL or $G$ biopsies. FG fibres were not observed in Sol muscle biopsies.

In control VL and $\mathrm{G}$ biopsies $(\mathrm{n}=3)$ all fibres displayed dark PAS staining intensity. One of the two control Sol biopsies, however, showed a weighted glycogen loss of $5 \%$ in the fast twitch fibres.

Glycogen is reported as the proportion of each

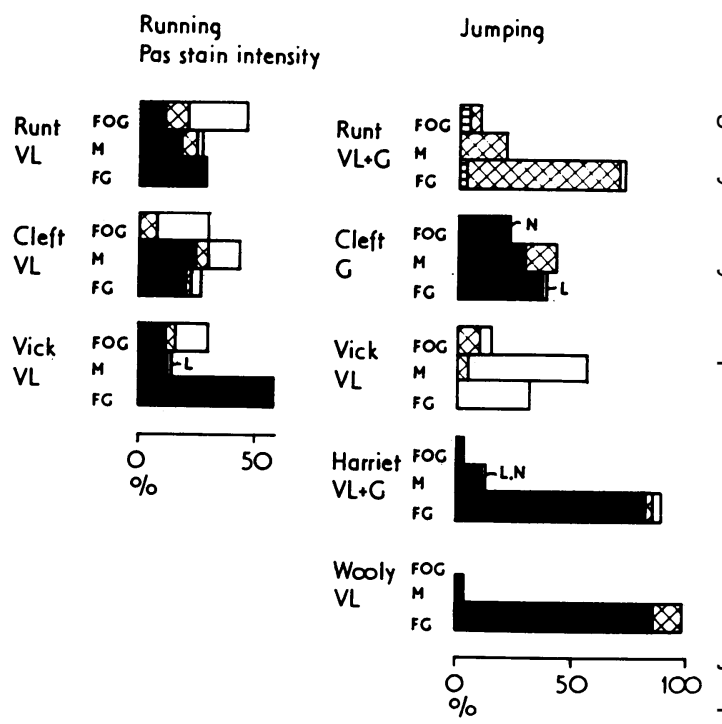

FIG. 1. Distribution of muscle glycogen loss in fast twitch fibres. Histograms of individual muscle biopsie separated according to exercise task, animal, an ${ }^{\infty}$ muscle. The length of each bar expresses fibre typ p percentage (sum of three bars equals $100 \%$ ). Shading reflects glycogen content. PAS stain intensit Dark $(D)$. 回 Moderate $(M)$. \# Light (L\&) Negative $(N)$.

$\%$ Weighted glycogen loss*

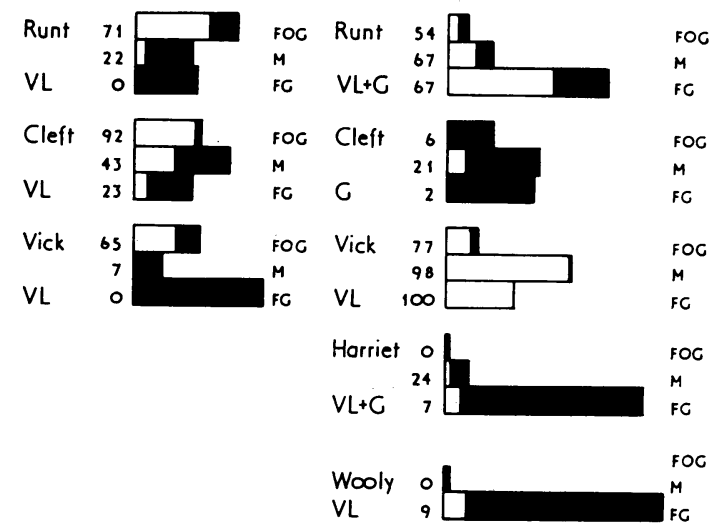

FIG. 2. Legend as in Fig. 1. * Light area represents $\%$ weighted glycogen depleted from that fibre type with bar representing total population of fibres. 


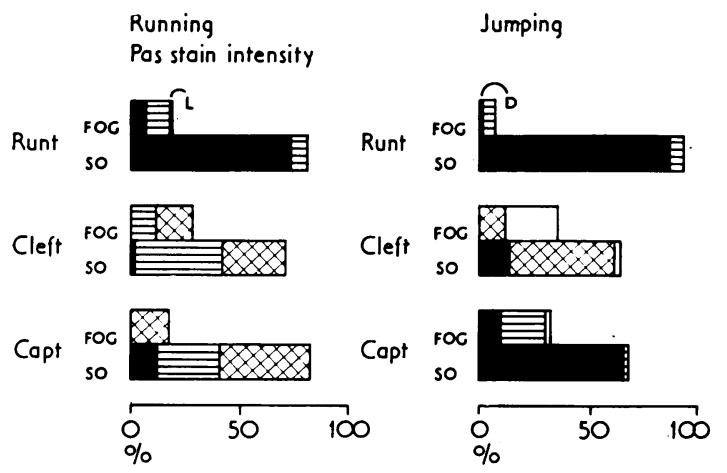

FIG. 3. Distribution of muscle glycogen loss in the soleus. Histograms of individual muscle biopsies separated according to exercise, animal, and muscle. The length of each bar represents fibre type percent within that biopsy (sum of both bars equals 100\%). Shading reflects glycogen content. PAS stain intensity.

$\square$ Dark (D). $⿴$ Moderate $(M) . \square$ Light $(L)$. $\square$ Negative $(N)$.

fibre type (FOG, M, FG, and SO fibres) that demonstrated dark, moderate, light, and negative PAS staining intensity. For example, in Runt's run VL biopsy, $21 \%, 23 \%$, and $56 \%$ of the FOG fibres stained dark, light, and negative respectively (Fig. 1). In all three VL run biopsies, the dark PAS staining fibres are predominantly FG and $M$ fibres. In two of these samples no evidence of depletion in FG fibres was observed. Thus, the lighter PAS staining or the apparently greatest glycogen loss was observed mainly in the FOG fibre population of run biopsies.

Having weighted the fibre PAS staining intensity from 3 to 0 (dark to negative), the loss of glycogen is expressed as the percent weighted glycogen loss within each fibre type (Fig. 2). From this calculation, it is seen that the greatest weighted glycogen loss with running occurs in the FOG fibres followed by some loss in the $M$. The least glycogen loss occurred in the FG fibre population. For example, in Runt's run VL, $71 \%, 22 \%$, and $0 \%$ of the glycogen was depleted from the FOG, M, and FG fibre populations, respectively.

Analysis of the PAS staining of fibres in jumping $\mathrm{VL}$ and $\mathrm{G}$ biopsies revealed a distinctly different pattern of glycogen loss than that of run biopsies (Fig. 1). In all five jump samples a

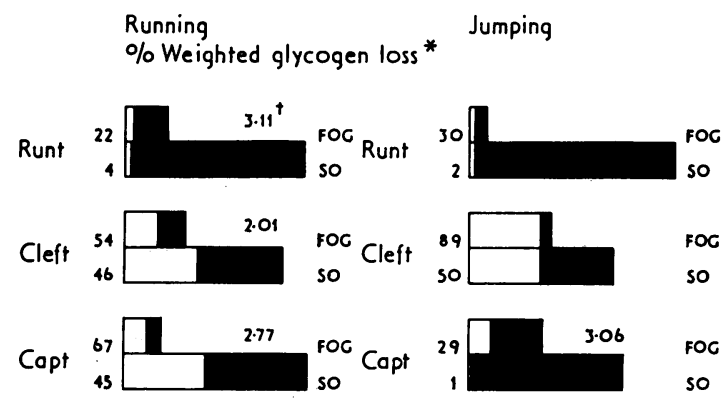

FIG. 4. Legend as in Fig. 3. * Light area represents $\%$ weighted glycogen depleted from that fibre type with bar representing total population of fibres. $\dagger$ Glycogen ( $\mathrm{mg} / \mathrm{lo0} \mathrm{g}$ wet muscle weight).

greater percentage of the FG or $\mathrm{M}$ fibres stained light or negative with PAS than the FOG fibres. That is, glycogen loss due to jumping was greatest in either the FG or $\mathrm{M}$ fibre populations. This is also evident from the percent weighted glycogen loss which was always highest in either FG or M fibre populations (Fig. 2).

Examination of the PAS staining in the Sol revealed essentially the same pattern of glycogen depletion whether running or jumping (Fig. 3). In all cases a greater percentage of the SO fibre population than the FOG stained dark with PAS. Conversely, a larger percent of the FOG fibres stained negative, light, or moderate with PAS. The occurrence of more glycogen depletion in the FOG fibre population than in the SO can also be readily identified from the percent weighted glycogen loss (Fig. 4). In Cleft's Sol run biopsy the smallest difference in glycogen depletion of FOG and SO fibres was observed. With this possible exception there is a clear selective glycogen depletion of the fast-twitch fibres of the Sol during both running and jumping.

\section{DISCUSSION}

There is substantial electrophysiological evidence which is consistent with the hypothesis that the recruitment of motor neurones is under conscious control and is related to the nature of the movement performed. For example, it has been demonstrated that the order of recruitment of motor units resulting from contractions 
elicited voluntarily and through tendon reflexes (tendon tap) and painful electrical stimuli of the plantar surface of the foot (polysynaptic) can be altered, although the order was usually constant (Ashworth et al., 1967). Grimby and Hannerz (1968) demonstrated that the recruitment order differed depending on the velocity of contraction as well as on proprioceptive afferent activity producing a facilitation of varying strength in different tonic units. Discordance of recruitment order between voluntary and reflex activation was found in about $20 \%$ of the motoneurones (Ashworth et al., 1967). Significant changes in recruitment order were produced in spinal subjects when the contraction stimulus was shifted from phasic to tonic reflexes. Tonic units were recruited first when activity was mediated through group Ia afferent fibres while phasic units fired initially when mediated through the pyramidal tract (Grimby and Hannerz, 1970). Similar results have been observed in intercostal muscles of the Gallus domesticus (Fedde et al., 1969). By increasing respired $\mathrm{CO}_{2}$ levels, phasic contractions were induced which were accompanied by an increase in frequency of small nerve action potentials and motor unit action potentials as well as selective recruitment of motor units with larger action potentials in nerve and muscle. But even in the light of this evidence, it is held by some that the order of recruitment of motor neurones is fixed depending on the stimulating $\triangle$ threshold which in turn is dictated by the size of $ळ$ the cell body. Arguments for and against have $\vec{\circ}$ been expressed recently (Granit and Burke, 1973).

Evidence for this size principal-threshold is

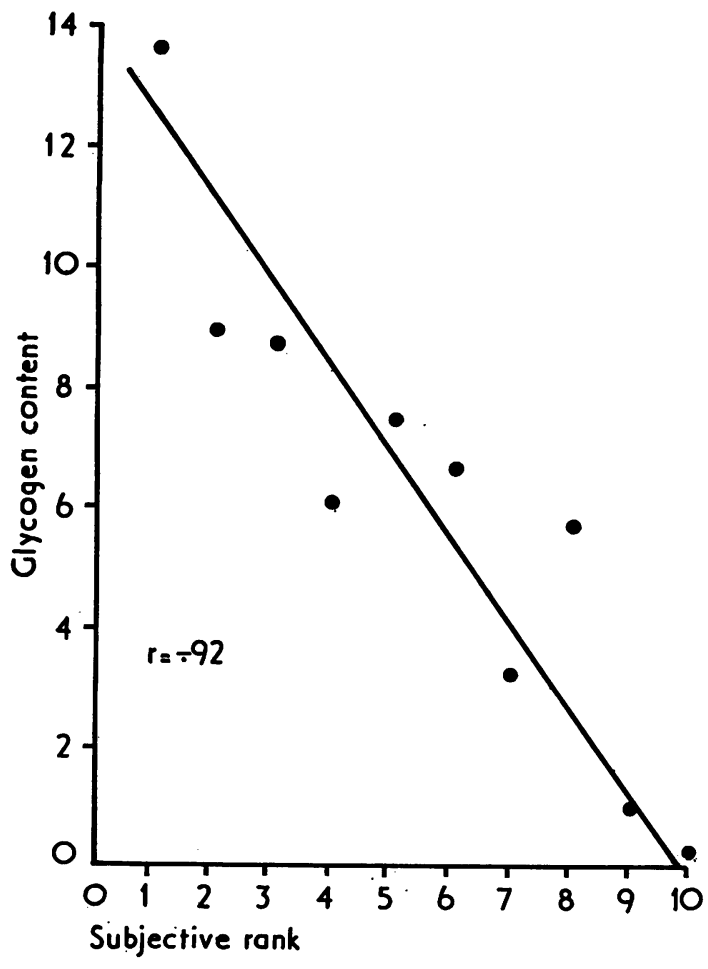

FIG. 5

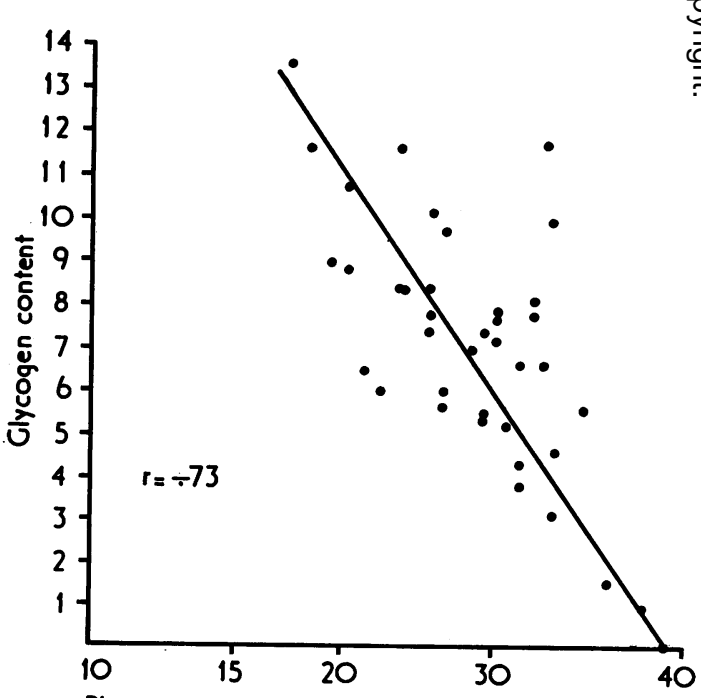

FIG. 6

FIGS 5 (left) and 6 (right). Correlations between biochemically determined glycogen content (mg glycogen/g tissue) and histochemical PAS staining intensity of serial pieces of the same biopsy. Figure 5 is the correlation between glycogen content and the subjective ranking of all the biopsies with respect to PAS staining intensity. Figure 6 correlates the glycogen content with the log-plot of microphotometric values of transmitted light intensities of the PAS sections. 
very convincing under the experimental conditions in which the data were collected-that is, in decerebrated cats (Henneman et al., 1965). But studies showing a flexible motoneuronal recruitment order clearly have been shown when the supraspinal influences on the motoneurone of the human were normal. The differences in the type of fibres that are depleted of glycogen in the bushbaby after running and jumping appear to be a reflection of this flexibility of motoneuronal recruitment. The consistent patterns of differential glycogen loss related to specific types of muscle fibres support the hypothesis that the motoneuronal recruitment pattern is not determined solely by motoneuronal size and that the bushbaby has the ability to utilize those motor units that are best suited to the movement desired. During a steady running pace, endurance is a more critical factor than tension, whereas in jumping movements high tension output is essential and, necessarily, endurance will be sacrificed.

The data shown in Figs 1-4 clearly demonstrate that the nature of the exercise dictates the specific types of muscle fibres that are depleted of glycogen after short bouts of exercise. In each animal after running FOG fibres of the VL and $G$ were preferentially depleted of glycogen relative to FG fibres, whereas either FG or 'moderate' fibres showed preferential depletion after brief bouts of jumping. In the Sol the performance of running and jumping results in an essentially similar pattern of glycogen loss preferentially in the fast-twitch fibres.

In control VL and $\mathrm{G}$ biopsies $(\mathrm{n}=3)$ all fibres had essentially the same PAS staining intensity and were all rated as dark. In one of the two control Sol samples, some of the FOG fibres stained moderate (2) with PAS as opposed to dark (3), whereas the other biopsy had a uniformly dark PAS staining intensity. The occurrence of some degree of glycogen depletion in FOG fibres has been observed previously in resting FOG muscles of guinea-pigs, rats, and mice and is thought to be indicative of the fibre recruitment involved in the animal's low-intensity movements in the cage. In the bushbabies, FOG depletion in control samples was observed only in the resting Sol, not in the resting VL and G.

To use glycogen loss, demonstrated histochemically, as an indicator of specific fibre recruitment, two conditions must be satisfied: first, that the PAS stain must be an adequate indicator of muscle glycogen and second, that the PAS stain must be reliably quantified. Figures 5 and 6 demonstrate that in previous work with guinea-pigs these two conditions are reasonably satisfied.

It has been assumed that muscle fibre glycogen loss is a reflection of muscle fibre recruitment in several previous studies that have dealt with the electrical stimulation (Kugelberg and Edström, 1968; Edgerton et al., 1970a; Burke et al., 1971). But, for glycogen loss to be employed as a meaningful indicator of fibre recruitment during exercise, it must be assumed that glycogen is the essential source of energy utilized during brief exercise bouts such as those studied here.

Muscle glycogen was shown to be reduced by $50 \%$ within 15 minutes after initiation of submaximal work in man and rats (Bergstrom and Hultman, 1967; Edgerton et al., 1973). The fact that glycogenolysis and glycolysis are activated within seconds of initiation of muscle work also indicates that glycogen is an immediate source of energy. Two trains of $6 / \mathrm{s}$ pulses convert $85 \%$ of a frog muscle sartorius total phosphorylase to phosphorylase $a$. (Danforth et al., 1962; Danforth and Helmreich, 1964). When glycogen is being degraded, phosphorylase is activated and glycogen synthetase activity is inhibited in vitro since glycogen degradation begins rapidly upon the onset of exercise. This strongly suggests that the muscle glycogen remaining after the brief bout of exercise used for the bushbabies was more a reflection of glycogenolysis than of glycogenesis. A muscle fibre's utilization of substrates other than glycogen for its energy also theoretically could negate glycogen loss as an indicator of motor unit recruitment patterns. The relative importance of blood glucose as a substrate that might affect the potential of glycogen depletion as an indicator of muscle fibre contractions is indicated by the fact that a maximal exercise for up to six minutes causes no change in blood glucose concentrations (Keul et al., 1967). Plasma free fatty acids also can serve as a very important energy source for working muscles (about $33 \%$ at a work rate of $200 \mathrm{~W}$ ) (Keul et al., 1972); the greater the duration of exercise, the greater its importance (Issekutz, 
RUNNING
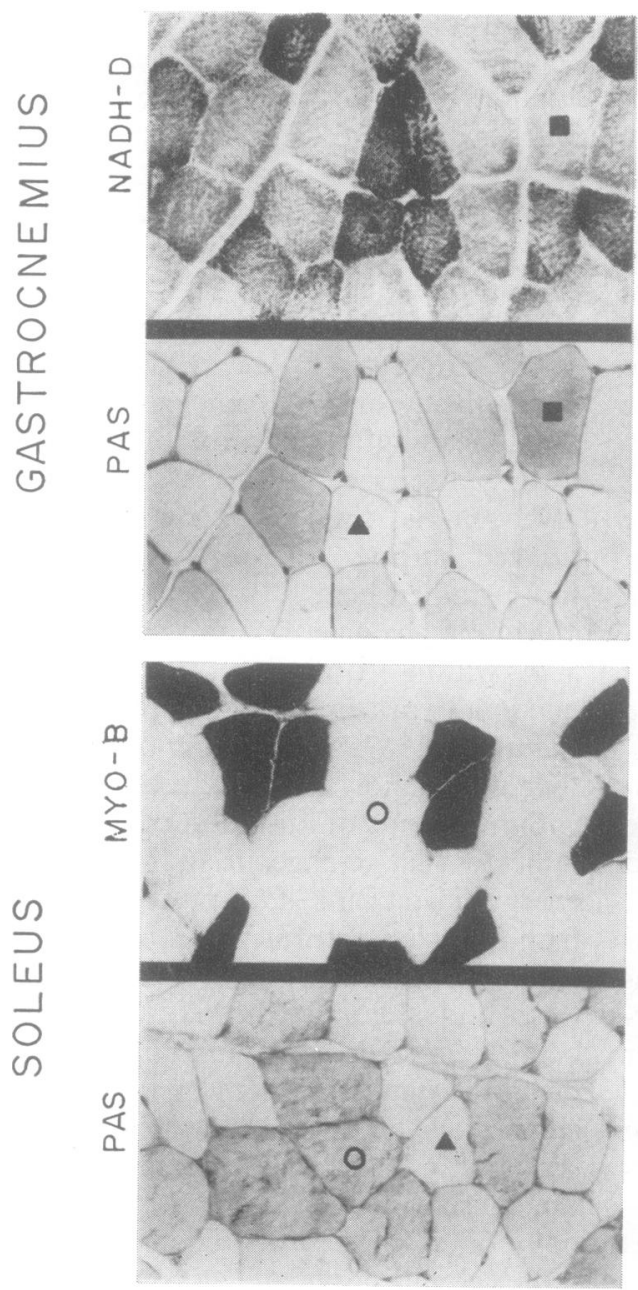

CONTROL

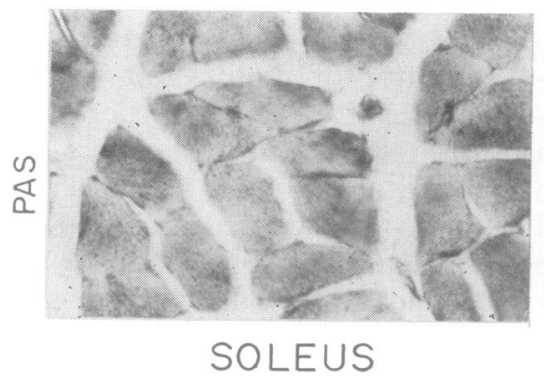

JUMPING
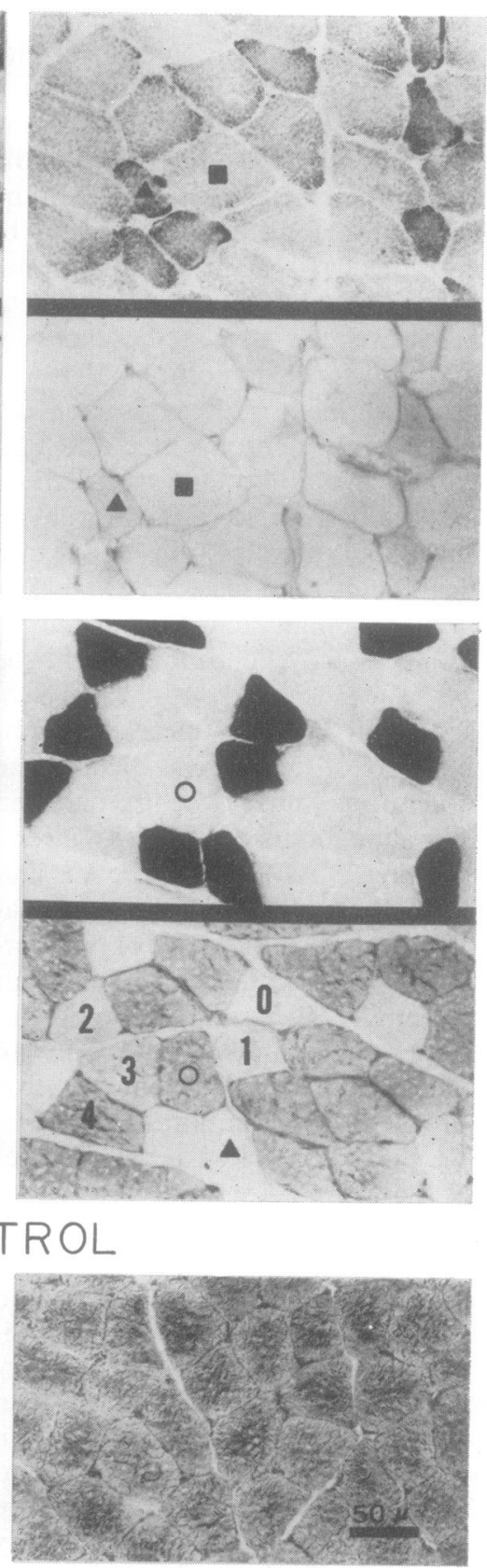

VASTUS LATERALIS

FIGURE 7. Serial histochemical sections of running and jumping exercise biopsies from fast-twitch muscle (gastrocnemius and vastus lateralis) and slow-twitch muscle (soleus). The stains represent oxidative capacity (NADH-D), glycogen content (PAS) and myosin ATPase activity (myo B). The fibre types are represented by a triangle (FOG), square (FG), and circle (SO). The numbers in the right photograph refer to a typical subjective quantification of the PAS staining intensity. 
1970). But its potential interference to the degree that FFA utilization camouflages glycogen degradation during a brief exercise is unlikely for several reasons. First, blood lactate at levels typical of moderate work intensities in humans $(50 \mathrm{mg} \%)$ causes a significant decrease in FFA mobilization and oxidation (Issekutz, 1970). Secondly, FFA oxidation is a more important energy source during a low-intensity exercise (running) than during high-intensity work (jumping). But, in spite of this, glycogen was clearly depleted after running in those muscle fibres that have the greatest blood supply and capacity to oxidize FFA (FOG). So, in effect, a clear differential pattern of glycogen loss was evident in spite of any slight camouflaging effects of other substrates that might be operative.

Different levels of glycogen after an exercise theoretically could result from different initial resting values. But there appears to be little difference between the glycogen content in FG and FOG fibres of the rested Galago. Some difference was observed between slow and fast twitch muscle fibre glycogen content of the Sol. Furthermore, in order to relate different glycogen depleted patterns to motoneuronal recruitment differences, it must be assumed that the amount of work performed or tension maintained per ATP was relatively constant. This seems a safe assumption when comparing fibre types with similar dynamic properties such as FG and FOG fibres but not when comparing fast twitch with slow twitch fibres. Goldspink et al. (1970) have reported greater efficiency of slow-twitch fibres in maintaining isometric tension while prepredominately fast twitch muscles performed more isotonic work per ATP than predominantly slow twitch muscles. Consequently, neither initial glycogen variations between FOG and FG fibres nor efficiency could account for the findings in this paper.

Could the differential patterns of glycogen loss be caused by differential hormonal responses to the exercise? Glycogenolysis is stimulated by catecholamines but the time course for plasma catecholamines release is slower than would be necessary to account for our differences in glycogen loss. In response to 40 minutes of a work load of about $70 \% \max \mathrm{VO}_{2}$ on a bicycle ergometer (Hartley et al., 1972) catecholamines are elevated in humans. In the frog sartorius the rate of change from phosphorylase $b$ to $a$ is 500 times greater at $30^{\circ} \mathrm{C}$ when induced by muscular contraction than by adrenaline (Danforth $e t$ al., 1962). But a hormonal explanation is even more unlikely when it is realized that the difference in the gly_ogen loss pattern between running and jumping would require a significant difference in the hormonal response or a difference in the sensitivity of each muscle fibre type depending on the kind of exercise. Neither of these events is likely to occur. But in spite of all of these potential metabolic limitations which would in all likelihood only make glycogen loss even less revealing of muscle fibre utilization than it is, a clear difference in the type of fibres that are glycogen depleted exists by changing the nature of movement (Fig. 7).

\section{SUMMARY}

Biopsies from the vastus lateralis, gastrocnemius, and soleus muscles of bushbabies after running and jumping were examined with respect to the glycogen loss from specific muscle fibre types. This was done as a means of determining the relative involvement of specific types of muscle fibres during slow continuous movement and a more rapid forceful movement.

First, it was found that the muscle glycogen content as measured spectrophotometrically was highly correlated with the PAS staining intensity of frozen tissue sections measured microphotometrically and by subjective ranking.

Secondly, it was observed that there was a greater loss of glycogen in the fast-twitch oxidative glycolytic than fast-twitch glycolytic fibres after running, whereas the opposite was true after jumping.

Thirdly, it was found that slow-twitch oxidative fibres compared with fast-twitch oxidative glycolytic fibres demonstrated more glycogen depletion after running, whereas the opposite selectivity was true after jumping.

These findings strongly suggest that the recruitment pattern of specific types of motor units is related to the nature of the specific movement being performed.

\section{REFERENCES}

Ashworth, B., Grimby, L., and Kugelberg, E. (1967). Comparison of voluntary and reflex activation of motor units. Journal of Neurology, Neurosurgery, and Psychiatry, 30, 91-98. 
Barnard, R. J., Edgerton, V. R., Furukawa, T., and Peter, J. B. (1971). Histochemical, biochemical, and contractile properties of red, white, and intermediate fibers. American Journal of Physiology, 220, 410-414.

Bergström, J., and Hultman, E. (1967). A study of the glycogen metabolism during exercise in man. Scandinavian Journal of Clinical and Laboratory Investigation, 19, 218228.

Burke, R. E., Levine, D. N., Zajac, F. E., III, Tsairis, P., and Engel, W. K. (1971). Mammalian motor units: physiological-histochemical correlation in three types in cat gastrocnemius. Science, 174, 709-712.

Danforth, W. H., and Helmreich, E. (1964). Regulation of glycolysis in muscle. I. The conversion of phosphorylase $b$ to phosphorylase $a$ in frog sartorius muscle. Journal of Biological Chemistry, 239, 3133-3138.

Danforth, W. H., Helmreich, E., and Cori, C. F. (1962). The effect of contraction and of epinephrine on the phosphorylase activity of frog sartorius muscle. Proceedings of the National Academy of Science of the United States of America, 48, 1191-1199.

Edgerton, V. R., Barnard, R. J., Peter, J. B., Gillespie, C. A., and Simpson, D. R. (1972a). Overloaded skeletal muscles of a nonhuman primate (Galago senegalensis). Experimental Neurology, 37, 322-339.

Edgerton, V. R., Barnard, R. J., Peter, J. B., Simpson, D. R., and Gillespie, C. A. (1970a). Response of muscle glycogen and phosphorylase to electrical stimulation in trained and nontrained guinea pigs. Experimental Neurology, 27, 46-56.

Edgerton, V. R., and Hewitt, H. (1972b). Relationship of twitch and metabolic properties of muscle fibers to recruitment patterns in various kinds of movements in animals. (Abstract.) Society for Neuroscience. Program and Abstracts. Second Annual Meeting, Houston, Texas, 1972 , p. 157.

Edgerton, V. R., and Lehto, S. (1972c). Utilization of skeletal muscle fiber types during exercise of a non-human primate. Medicine and Science in Sports, 4, 50.

Edgerton, V. R., Saltin, B., Essen, B., and Simpson, D. R. (1974). Glycogen depletion in specific types of human skeletal muscle fibers after various work routines. In Second International Symposium on Biochemistry of Exercise. Edited by Howald, H. (In press.)

Edgerton, V. R., and Simpson, D. R. (1969). The intermediate muscle fiber of rats and guinea pigs. Journal of Histochemistry and Cytochemistry, 17, 828-838.

Edgerton, V. R., Simpson, D. R., Barnard, R. J., and Peter, J. B. (1970b). Phosphorylase activity in acutely exercised muscle. Nature, 225, 866-867.

Edgerton, V. R., Simpson, D. R., and Gillespie, C. A. (1973). Functional implications of muscle fibre types as evidenced by muscle glycogen at rest, after exercise, and electrical stimulation. In Physical Fitness-Proceedings of a Satellite Symposium of the 25th International Congress of Physiological Sciences, Prague, 1971, pp. 148-153. Edited by V. Seliger. Universita Karlova: Prague.

Fedde, M. R., DeWet, P. D., and Kitchell, R. L. (1969). Motor unit recruitment pattern and tonic activity in respiratory muscles of Gallus domesticus. Journal of Neurophysiology, 32, 995-1004.
Goldspink, G., Larson, R. E., and Davies, R. E. (1970). The immediate energy supply and the cost of maintenance of isometric tension for different muscles in the hamster. Zeitschrift fuer vergleichende Physiologie, 66, 389-397.

Granit, R., and Burke, R. E. (1973). The control of movement and posture. Brain Research, 53, 1-28.

Grimby, L., and Hannerz, J. (1968). Recruitment order of motor units on voluntary contraction: changes induced by proprioceptive afferent activity. Journal of Neurology, Neurosurgery, and Psychiatry, 31, 565-573.

Grimby, L., and Hannerz, J. (1970). Differences in recruit- ment order of motor units in phasic and tonic flexion reflex in 'spinal man'. Journal of Neurology, Neurosurgery, and Psychiatry, 33, 562-570.

Guth, L., and Samaha, F. J. (1969). Qualitative differences between actomyosin ATPase of slow and fast mammalian muscle. Experimental Neurology, 25, 138-152.

Hartley, L. H., Mason, J. W., Hogan, R. P., Jones, L. G., $\unrhd$ Kotchen, T. A., Mougey, E. H., Wherry, F. E., Pennington, ô L. L., and Ricketts, P. T. (1972). Multiple hormonal responses to prolonged exercise in relation to physical $\overrightarrow{0}$ training. Journal of Applied Physiology, 33, 607-610.

Henneman, E., Somjen, G., and Carpenter, D. O. (1965). $\vec{\omega}$ Excitability and inhibitibility of motoneurons of different sizes. Journal of Neurophysiology, 28, 599-620.

Huijing, F. (1970). A rapid enzymic method for glycogen estimation in very small tissue samples. Clinica Chimica Acta, 30, 567-572.

Issekutz, B., Jr (1970). Interrelationships of free fatty acidg, $\infty$ lactic acid, and glucose in muscle metabolism. In Physiolo and Biochemistry of Muscle as a Food. 2, pp. 623-648 Edited by E. J. Briskey, R. G. Cassens, and B. B. Marsh. University of Wisconsin Press: Madison.

Keul, J., Doll, E., and Keppler, D. (1967). The substratec supply of the human skeletal muscle at rest, during agid after work. Experientia, 23, 974-979.

Keul, J., Doll, E., and Keppler, D. (1972). Energy Metabolidìn of Human Muscle. Medicine and Sport, vol. 7, p. 1\%. Karger: Basel.

Kugelberg, E., and Edström, L. (1968). Differential histo-O chemical effects of muscle contractions on phosphorylase and glycogen in various types of fibres: relation to fatigue. Journal of Neurology, Neurosurgery, and Psychiatry, 31, 415-423.

Novikoff, A. B., Shin, W. Y., and Drucker, J. (1961). Mitochondrial localization of oxidative enzymes. Staining $\overline{\bar{O}}$ results with two tetrazolium salts. Journal of Biophysical 3 and Biochemical Cytology, 9, 47-61.

Pearse, A. G. E. (1960). Histochemistry-Theoretical and Applied. 2nd edn, pp. 832, 854, 899. Churchill: London.

Peter, J. B., Barnard, R. J., Edgerton, V. R., Gillespie, C. A., and Stempel, K. E. (1972). Metabolic profiles of three? fiber types of skeletal muscle in guinea pigs and rabbits. Biochemistry, 11, 2627-2633.

Wattenberg, L. W., and Leong, J. L. (1960). Effects of coenzyme Q10 and menadione on succinate dehydrogenase activity as measured by tetrazolium salt reduction. Journal of Histochemistry and Cytochemistry, 8, 296-303. 